\title{
Berberine in Combination with Insulin Has Additive Effects on Titanium Implants Osseointegration in Diabetes Mellitus Rats
}

\author{
Li Lu, Huang Zhijian, Li Lei, Chen Wenchuan, and Zhu Zhimin \\ Department of Prosthodontics, West China Hospital of Stomatology, State Key Laboratory of Oral Diseases, Sichuan University, \\ Chengdu 610041, China
}

Correspondence should be addressed to Chen Wenchuan; hxkqcwc@scu.edu.cn and Zhu Zhimin; zzhimin@163.com

Received 9 September 2015; Revised 9 November 2015; Accepted 25 November 2015

Academic Editor: Bhushan Patwardhan

Copyright (C) $2015 \mathrm{Li} \mathrm{Lu}$ et al. This is an open access article distributed under the Creative Commons Attribution License, which permits unrestricted use, distribution, and reproduction in any medium, provided the original work is properly cited.

\begin{abstract}
This study evaluated the effects of berberine in combination with insulin on early osseointegration of implants in diabetic rats. Fifty male Sprague-Dawley rats were randomly divided into 5 groups: healthy rats were used as control (HC), and streptozotocininduced diabetic rats were treated with insulin, berberine, berberine + insulin (IB), or no treatment. Each rat received one machined-surface cp-Ti implant into the right tibia and was given insulin injection and/or gavage feeding with berberine daily for 8 weeks until being sacrificed. Serum levels of alkaline phosphatase (ALP) and bone gamma-carboxyglutamic acid-containing protein (BGP) were analyzed in each group. Peri-implant mineral apposition was marked by fluorochrome double-labeling and osseointegration was histomorphologically examined. The ALP and BGP levels decreased in diabetic rats but were successfully corrected by insulin and berberine combined treatment. Moreover, untreated diabetic rats had less labeled mineral apposition and impaired osseointegration. In contrast, Groups I, B, and IB were observed with increased peri-implant bone formation. The combination treatment of insulin and berberine was more effective than each administrated as a monotherapy. These results suggest that berberine combined with insulin could promote osseointegration in diabetic rats, thereby highlighting its potential application to patients, though further studies are needed.
\end{abstract}

\section{Introduction}

"Osseointegration" refers to the formation process of a direct interface between an implant and living bone [1]. Thereafter, implant has been extensively studied and widely used in clinics to replace missing teeth and restore oral functions. However, many systemic diseases such as osteoporosis, diabetes, and autoimmune diseases may interfere with the implant osseointegration in clinical practice [2]. Studies have shown that diabetes caused a higher failure rate of implants and poorer bone implant integration $[3,4]$. Type 2 diabetes mellitus (T2DM), the most common form of diabetes mellitus, is a metabolic disorder characterized by hyperglycemia resulting from peripheral insulin resistance with defective insulin secretion. T2DM is also associated with systemic chronic progressive disorders in kidneys, nerves, blood vessels, bones, and other tissues, which can lead to diminished immune response and increased inflammatory effect $[5,6]$. Also, substances such as advanced glycation end products (AGEs) and cytokines have deleterious effects on osteoblasts and the bone marrow microenvironment [7]. All of the systemic changes above would impair the osseointegration, as well as the long-term function of dental implants.

It is accepted that survival of implant can be improved when plasma glucose level is brought under control [8]. However, others admit that insulin alone is insufficient to reverse all the negative impact of diabetes on bone healing, with impaired bone implant integration in both animal models and patients [9-11]. One explanation may be the impossibility of continuously monitoring glucose and automatically adjusting insulin delivery; another important reason may lie in insulin resistance. Hence, there is a considerable interest in seeking complementary and alternative approaches in improving implant success in diabetes, especially orally available drugs which could mimic insulin action and somehow overcome insulin resistance [12]. 
Berberine ( $\left.\mathrm{BBR}, \mathrm{C}_{20} \mathrm{H}_{18} \mathrm{NO}_{4}\right)$ is an isoquinoline alkaloid purified from herbal plants including Coptis chinensis, Hydrastis canadensis, and Berberis aquifolium [13]. This naturally occurring molecule displays a broad array of pharmacological effects beneficial to a number of diseases. For example, it has been used as a nonprescription drug for treating infectious diseases like diarrhea in Asia for centuries. The hypoglycemic effect of berberine was first discovered in 1988 by accident when diabetic patients were found to have lowered serum glucose level with berberine playing its antidiarrheal role [14]. Thereafter, more studies have shown that berberine could potently augment glucose uptake into adipose and muscle tissues through multiple mechanisms, including insulin-dependent PTP1B, insulinindependent AMPK, increasing the affinity of low-activity glucose transporters (gluT1), and improving insulin sensitivity [15-17]. In STZ-induced diabetic rats, berberine potently lowered rats fasting blood glucose levels and improved oral glucose tolerance. Additionally, berberine also lowered multiple factors related to insulin resistance including blood cholesterol and triacylglycerols levels [18]. In diabetic patients, the hypoglycemic effect of berberine was similar to that of metformin [19]. In another aspect, berberine has been reported to promote osteogenic differentiation of bone marrow-derived mesenchymal stem cells (MSCs) through canonical Wnt $/ \beta$-catenin signaling [20]. Also, osteogenic genes expression, including osteopontin, osteocalcin, and Runx2 (Runt-related transcription factor 2), was upregulated in osteoblasts by berberine [21]. Furthermore, berberine inhibited osteoclasts activity through suppressing the NFkappaB and Akt pathways [22, 23]. In vivo, osteoporosis in both glucocorticoid-induced rat model and senescence accelerated mice P6 (SAMP6) was prevented by berberine $[24,25]$. Since functions of berberine in glucose homeostasis, insulin sensitizing, and bone anabolism are closely related to the promotion of bone formation in diabetic patients, we therefore hypothesize that berberine alone and in combination with insulin therapy may be beneficial to the implant osseointegration in T2DM. The combination therapy seems to be more promising for the different and complementary mechanisms of action of berberin and insulin.

\section{Experimental Section}

2.1. Experimental Implants. The implants were provided by Professor Liu Xiaoguang (the National Engineering Research Center of Biomaterials, Sichuan University, Chengdu, China). The cp-Ti implants are $4 \mathrm{~mm}$ in length and $1 \mathrm{~mm}$ in diameter with machined surface. All implants were washed with deionized water in the ultrasonic bath and sterilized in an autoclave before surgery.

2.2. Experimental Animals. This experiment protocol was approved by the Animal Research Bioethics Committee of Sichuan University and was conducted following the international animal welfare standards. A total of 50 male SpragueDawley (SD) rats, provided by the Experimental Animal Center at Sichuan University, were selected for study. They were 8 weeks of age, weighing around $190 \mathrm{~g}$. Every two rats were housed in each cage in a 12-hour day/night cycle and had free access to water and rat normal pellet diet (NPD, DaShuo Laboratory Animal Co., Ltd., Chengdu, China).

After 1 week of acclimation (start of the study), the rats were randomly assigned into 5 groups ( $n=10$ per group): (1) healthy rats without treatment (Group HC); (2) diabetic rats without treatment (Group D); (3) diabetic rats treated with insulin (Group I); (4) diabetic rats treated with berberine (Group B); (5) diabetic rats treated with both insulin and berberine (Group IB). Type 2 diabetes rat model was built according to the combination of high-fat diet-fed and lowdose streptozotocin-treatment method [26]. Four diabetic groups were fed with high-fat diet (HFD, 58\% total kcal of fat, $25 \%$ of protein, and $17 \%$ of carbohydrate) while Group $\mathrm{HC}$ were continually fed with NPD. After 2 weeks of dietary manipulation, the high-fat diet rats (i.e., Groups D, I, B, and IB) were given intraperitoneally $35 \mathrm{mg} / \mathrm{kg}$ freshly prepared streptozotocin (STZ, Sigma, St. Louis, MO, USA), while the healthy control rats (Group HC) were injected with $1 \mathrm{~mL}$ of vehicle citrate buffer ( $\mathrm{pH} 4.4)$. The plasma glucose levels (PGLs) were measured 72 hours after the STZ administration and rats with nonfasting PGLs higher than $16.7 \mathrm{mmol} / \mathrm{L}$ threshold were considered to be diabetic and chosen for further studies. All the rats were continually fed on their respective diets until being sacrificed.

2.3. Implant Surgery. After diabetic rat model establishment (0 days), animals were anesthetized intraperitoneally $(10 \%$ chloral hydrate, $3 \mathrm{~mL} / \mathrm{kg}$ ). The surgical area was shaved and disinfected with $75 \%$ ethanol. Then, the implant bed was prepared by standardized drilling procedure with saline irrigation [27], followed by inserting the implant perpendicularly to the long axis of the right tibia in distal tibia metaphysis ( $2 \mathrm{~mm}$ distal to the proximal growth plate) (Figure 1). After surgery, the skins were carefully and separately sutured to ensure the submerged healing of implants with no functional loading. All animals received the intramuscular antibiotic prophylaxis in the first three days after surgery.

2.4. Pharmaceutical Treatment. Group I rats received a daily subcutaneous injection of 1-2 UI NPH insulin (Humulin N, Lilly, Fegersheim, France) for 8 weeks according to literature review [28]. Based on prior study, Group B received daily $300 \mathrm{mg} / \mathrm{kg}$ of berberine (Sigma, St. Louis, MO, USA) dissolved in $0.5 \%$ carboxymethyl cellulose via oral gavage [29]. Group IB received insulin and berberine with doses and methods similar to those of Groups I and B. Group HC received a daily gavage of saline solution. A summary of the animal grouping and treatments is presented in Figure 2.

2.5. Fluorochrome Double-Labeling. Two fluorochromes were injected sequentially: alizarin red $(25 \mathrm{mg} / \mathrm{kg} / \mathrm{im}$, Sigma, St. Louis, MO, USA) at 1 week after surgery and calcein green (30 mg/kg/im, Sigma, St. Louis, MO, USA) at 7 weeks after surgery. Both fluorochrome labels could bind to the sites of active bone deposition shortly after injection. This enabled 


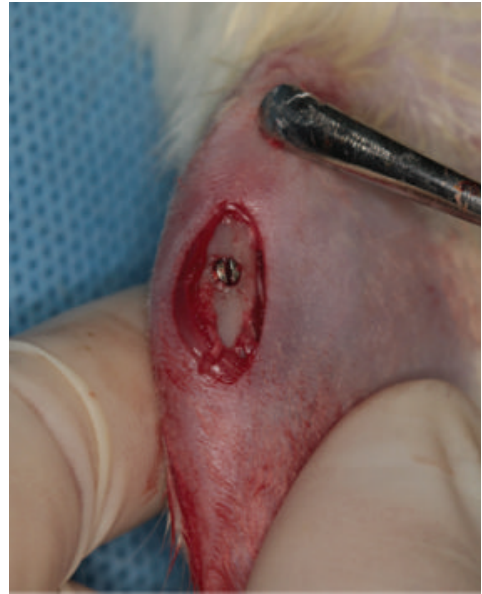

(a)

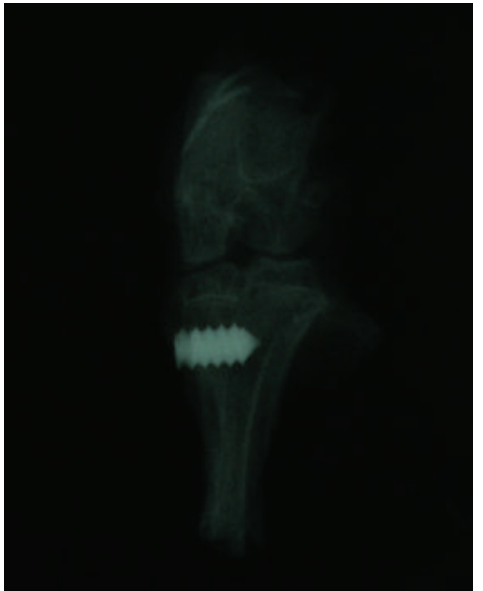

(b)

FIGURE 1: Implant surgery and postsurgery radiograph. (a) The implant was placed into the hole prepared in the tibia metaphysis. (b) Radiograph of tibia with implant after surgery.

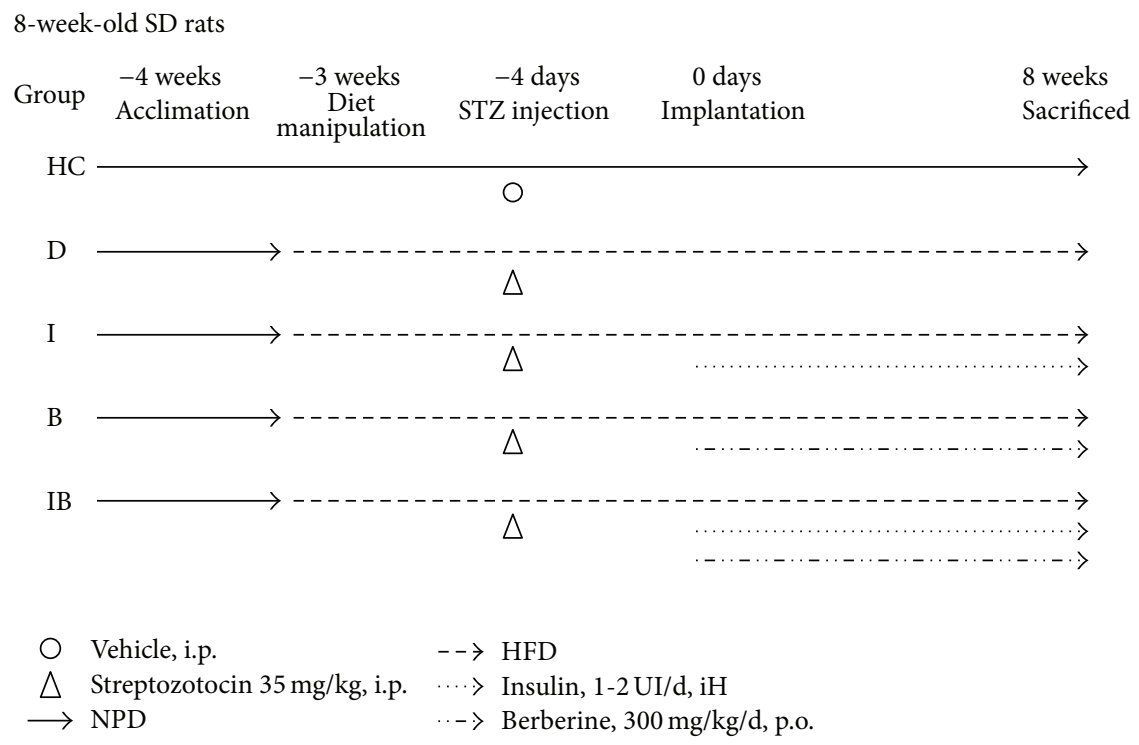

FIGURE 2: Summary of animal grouping and treatments. Animals were randomly allocated into five groups: healthy rats as control (HC) and 4 groups of diabetic rats including (1) no additional treatment, (2) insulin, (3) berberine, and (4) berberine + insulin therapies; the treatments were given to animals for 8 weeks until being sacrificed.

the identification of bone deposition around implants at different time points.

2.6. Measuring of Blood Glucose and Body Weight. Blood samples were randomly obtained from the animals by tail snipping, with plasma glucose levels (PGLs) measuring by an Accu-Chek glucose meter (Roche Diagnostics, Laval, QC, Canada). PGLs and body weights of the rats $(n=10$ specimens per group) were measured at -3 weeks, 0 days, 1 week, 4 weeks, and 8 weeks, respectively.

2.7. Serum Biochemical Assays. All the animals were sacrificed 8 weeks after surgery with a lethal dose of pentobarbital.
Blood samples ( $n=10$ specimens per group) were collected at the time of sacrifice and analyzed for serum alkaline phosphatase (ALP) and bone gamma-carboxyglutamic acidcontaining (also known as osteocalcin) protein (BGP). Serum values of BGP were measured by rat osteocalcin EnzymeLinked Immunosorbent Assay (ELISA) Kit (DRG International Inc., NJ, USA). ALP activity was analyzed using a pNPP Alkaline Phosphatase Assay Kit (AnaSpec Inc., CA, USA).

2.8. Fluorochrome Double-Labeling Analysis. The tibias with implants ( $n=10$ specimens per group) were harvested after the removal of soft tissue and maintained in a $4 \%$ neutral-buffered solution of formalin at $4^{\circ} \mathrm{C}$ for fixation. 
TABLe 1: Plasma glucose levels ( $\mathrm{mmol} / \mathrm{L})$ of rats during the whole study.

\begin{tabular}{|c|c|c|c|c|c|}
\hline Animal status & -3 weeks & 0 days & 1 week & 4 weeks & 8 weeks \\
\hline Group HC & $5.8 \pm 0.7^{1}$ & $6.4 \pm 0.8^{1}$ & $6.3 \pm 0.8^{1}$ & $6.6 \pm 0.8^{1}$ & $6.7 \pm 0.7^{1}$ \\
\hline Group D & $6.2 \pm 0.7^{1}$ & $27.1 \pm 2.2^{2}$ & $24.0 \pm 2.4^{5}$ & $29.1 \pm 1.9^{3}$ & $25.4 \pm 1.2^{3}$ \\
\hline Group I & $6.1 \pm 0.8^{1}$ & $26.8 \pm 3.0^{2}$ & $11.2 \pm 1.8^{3}$ & $8.7 \pm 1.2^{1}$ & $7.1 \pm 0.6^{1}$ \\
\hline Group B & $6.2 \pm 0.7^{1}$ & $26.8 \pm 3.7^{2}$ & $20.1 \pm 1.6^{4}$ & $17.5 \pm 2.2^{2}$ & $11.2 \pm 1.3^{2}$ \\
\hline Group IB & $6.3 \pm 0.6^{1}$ & $24.4 \pm 1.4^{2}$ & $8.7 \pm 1.7^{2}$ & $6.4 \pm 1.2^{1}$ & $6.7 \pm 0.9^{1}$ \\
\hline
\end{tabular}

Means with identical superscripts in a given column are not statistically significant at the $5 \%$ alpha level.

TABLE 2: Weights (g) of rats during the whole study.

\begin{tabular}{|c|c|c|c|c|c|}
\hline Animal status & -3 weeks & 0 days & 1 week & 4 weeks & 8 weeks \\
\hline Group HC & $207 \pm 2.8^{1}$ & $262 \pm 21.9^{1}$ & $275 \pm 20.3^{1}$ & $344 \pm 19.7^{2}$ & $424 \pm 20.0^{3}$ \\
\hline Group D & $212 \pm 2.9^{1}$ & $314 \pm 15.4^{2}$ & $291 \pm 13.8^{1}$ & $289 \pm 18.4^{1}$ & $267 \pm 18.1^{1}$ \\
\hline Group I & $209 \pm 3.9^{1}$ & $323 \pm 30.1^{2}$ & $301 \pm 19.5^{1}$ & $308 \pm 23.4^{2}$ & $374 \pm 30.6^{2}$ \\
\hline Group B & $212 \pm 5.3^{1}$ & $314 \pm 29.4^{2}$ & $302 \pm 25.4^{1}$ & $284 \pm 28.9^{1}$ & $277 \pm 27.7^{1}$ \\
\hline Group IB & $210 \pm 3.9^{1}$ & $317 \pm 20.7^{2}$ & $307 \pm 26.8^{1}$ & $313 \pm 30.0^{2}$ & $397 \pm 27.9^{2}$ \\
\hline
\end{tabular}

Means with identical superscripts in a given column are not statistically significant at the $5 \%$ alpha level.

The specimens were dehydrated in an ascending series of alcohol concentrations and embedded with methyl methacrylate. Afterwards, the samples were sectioned along the longitudinal direction of the implant by a rotary diamond saw (SP1600/2600, Leica, Nussloch, Germany) and each tibia specimen could get 3 to 4 slides, which were ground to a final thickness of approximately $30 \mu \mathrm{m}$ by a microtome (Leica SP2600 Ultramiller, Nussloch, Germany). The slides were first observed under a Nikon Eclipse 300 fluorescence microscope (Compix Inc., Sewickley, PA, USA) to determine the mineral apposition at different time points.

2.9. Histomorphological Analysis. After the labeling analysis, the slides with section through the central part of screw in each tibia specimen were selected and stained with $1 \%$ toluidine blue ( $n=10$ slides per group). Images were captured through a microscope (DXM 1200, Nikon, Tokyo, Japan) and evaluated using a computerized image analyzer (Image Pro Plus V6.0, Media Cybernetics, Bethesda, MD, USA). Values of bone-to-implant contact ratio (BIC, the percentage of linear surface of the implant directly contacted by mineralized bone) were measured along the whole implant surface and bone area fraction occupancy (BAFO, the percentage of bone filling the spaces between implant threads) was evaluated in the spaces within the whole implant threads [30].

2.10. Statistical Analysis. All of the experiments were repeated three times, and means \pm SDs (standard deviations) were calculated for each array of treatments for preliminary numerical comparisons. Intergroup differences were first analyzed using one-way ANOVA followed by Fisher's Least Significant Difference (LSD) post hoc tests at a significance level of $\alpha=0.05$. All data was analyzed with SPSS 19.0 software (SPSS, Inc., Chicago, USA).

\section{Results}

At 8 weeks postoperatively, all the surgery sites showed adequate healing, with no implant loss or infection.

3.1. Plasma Glucose Levels. Plasma glucose levels were presented in Table 1. Before STZ administration, the average of PGLs in each treatment group ranged from 5.8 to $6.3 \mathrm{mmol} / \mathrm{L}$, considered to be normal in this study. After STZ administration, PGLs in the diabetic rats showed a significant increase. After treatment, both insulin and/or berberine effectively lowered the PGLs. At the end of the study, Groups I and IB had PGLs restored to their prestudy levels. Berberine moderately lowered PGLs to $11.2 \mathrm{mmol} / \mathrm{L}$.

3.2. Body Weight. Table 2 shows the fluctuation of animal weights. After diet manipulation, animals fed with HFD weighed significantly greater than those fed with NPD $(P<$ 0.05). However, the induction of diabetes caused a rapid loss of weights. At the end of study, DM rats in Groups I and IB gained weight; nevertheless, rats in Groups D and B continued to lose weight.

3.3. Plasma Levels of BGP and ALP. Figure 3 showed that, after 8 weeks, insulin and/or berberine substantially increased the plasma BGP levels compared to that of Group D. Moreover, the plasma BGP levels in Group IB were significantly higher than that in Group $\mathrm{HC}(P<0.05)$. As for the plasma ALP levels, the values in Groups B and IB seem to be higher than Group D; the differences were not significant $(P>0.05)$.

3.4. Fluorescence Observations. Figure 4 showed mineral appositions around the implant, which was determined by fluorochrome double-labeling. The areas in green or red 

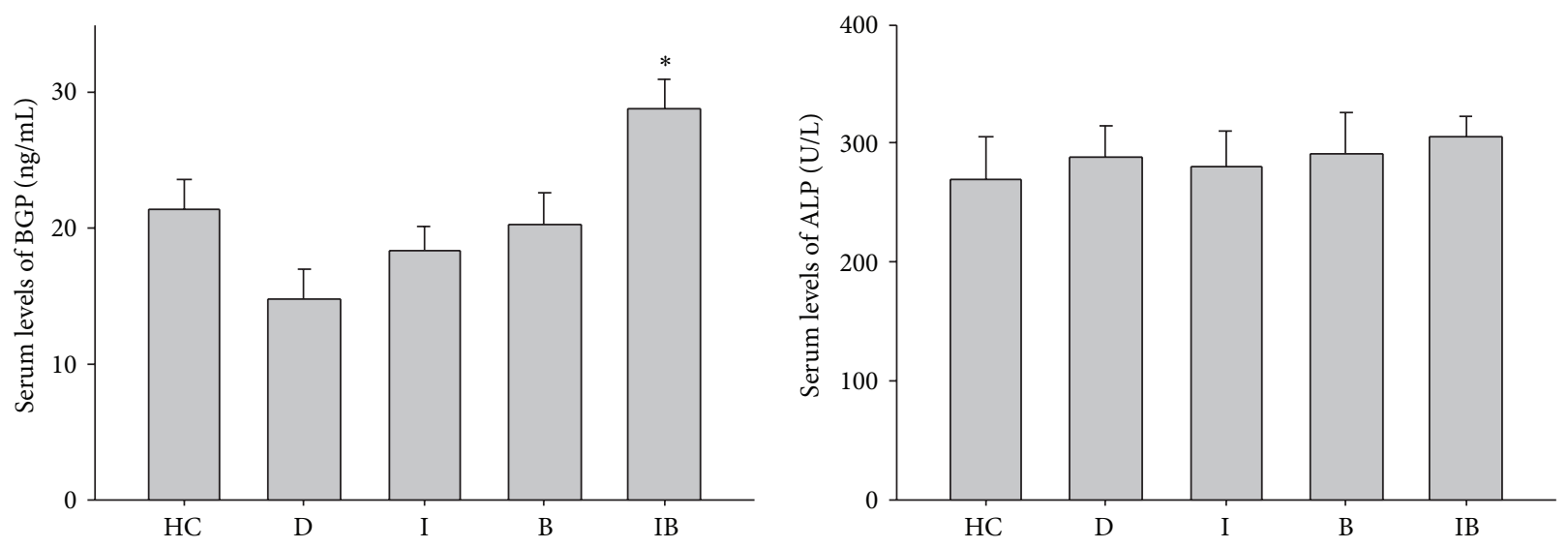

Figure 3: Plasma BGP and ALP levels at 8 weeks after implantation. ${ }^{*} P<0.05$, for Group HC versus Group IB.
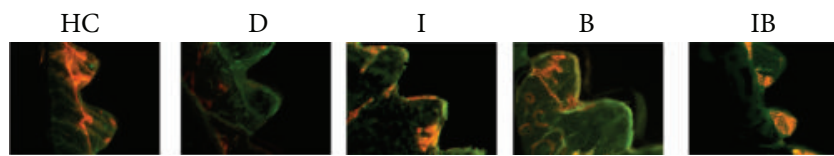

FIGURE 4: Fluorochrome double-labeling images of peri-implant osseointegration under microscopy at 10x (HC: healthy control; D: nontreated diabetic; I: insulin-treated diabetic; B: berberine-treated diabetic; IB: berberine + insulin-treated diabetic). Red was labeled by alizarin red at week 1 after surgery and green was labeled by calcein green at week 7 after surgery.

represented regions of calcium precipitation labeled by fluorochromes during the healing phases: red being at week 1 and green at week 7 after surgery. All groups but the diabetic exhibited obvious red labeling: larger scales of red labeling were observed in Groups HC and IB, whereas only a few red spots were observed in Group D. In addition, only faint green labeling was observed in all groups.

3.5. Histomorphometric Analyses. Figure 5(a) showed bone formation around implants. Comparison of bone filling the spaces between threads: there was the least osseointegration (discontinuance bone, minor direct contact) in Group D and the most osseointegration (mostly lamellar bone, dense and well organized, major contact) in Groups $\mathrm{HC}$ and IB. In quantitative analysis (Figures 5(b) and 5(c)), insulin increased BIC 1.7-fold and BAFO 1.3-fold compared with Group D. Berberine increased BIC 1.3-fold and slightly increased BAFO. The BIC and BAFO were successfully restored to normal level after insulin and berberine combined treatment (no significant differences compared with those of Group HC $(P>0.05))$.

\section{Discussion}

Diabetes mellitus has been widely studied for its influence on bone healing and remodeling and is shown to be detrimental to dental implants osseointegration. Consistent with previous studies [11], impaired new bone formation (little fluorochrome labeling) and poor osseointegration (decreased $\mathrm{BIC}$ and BAFO values) were observed in the untreated diabetic rats.

Previous studies have mainly discussed application of insulin to control serum glucose and improve implant osseointegration. However, the effects remained controversial due to the heterogeneity of diabetic models and the methodologies of insulin therapy used in their studies. The diabetic rat model in this study was induced by two steps: first, high-fat chow feeding to induce obesity, compensatory hyperinsulinemia, and insulin resistance, and second, the administration of low-dose STZ to destroy a part of pancreatic beta cells, leading to a reduction of insulin in serum and causing hyperglycemia. This STZ model is a widely used model in type 2 diabetic research that replicates metabolic characteristics of the human syndrome [26]. In this study, the changes of plasma glucose levels and weight substantiated onset of diabetic symptoms. The rats in Group $\mathrm{D}$ had significantly higher PGLs and increased body weight loss. The diabetic animals treated by insulin had their PGLs gradually restored to the normal range, indicating continuous regulation of plasma glucose levels in this study, which would avoid the major side effect of hypoglycemia usually seen in an intensive insulin therapy [31]. In fact, the fear of hypoglycemia commonly complicated the effective glycemic control both in patients and in physicians [32]. In this study, insulin only moderately increased BIC and BAFO, which were not comparable to the healthy controls. This might contribute to the fact that the body cells could not use insulin effectively due to insulin resistance (defects in insulinrelated signaling). Our study showed that insulin could not counterbalance all the negative changes caused by diabetes, which was in agreement with former studies showing that the $\mathrm{BIC}$ and removal torque were significantly lower compared to the healthy controls $[9,10]$.

Berberine has been recently reported for its dual functions in boosting bone remodeling and maintaining glucose homeostasis. Consistent with previous observations, the administration of berberine alone could effectively lower PGL but failed to restore it to the normal level [18]. The explanation might be that although berberine could activate 

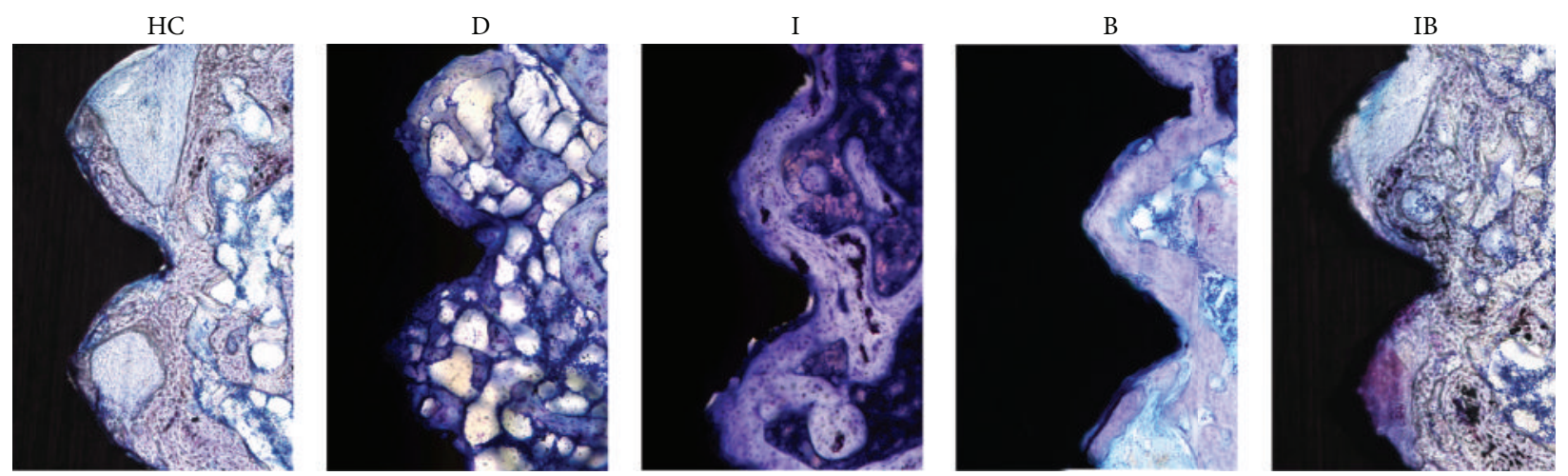

(a)

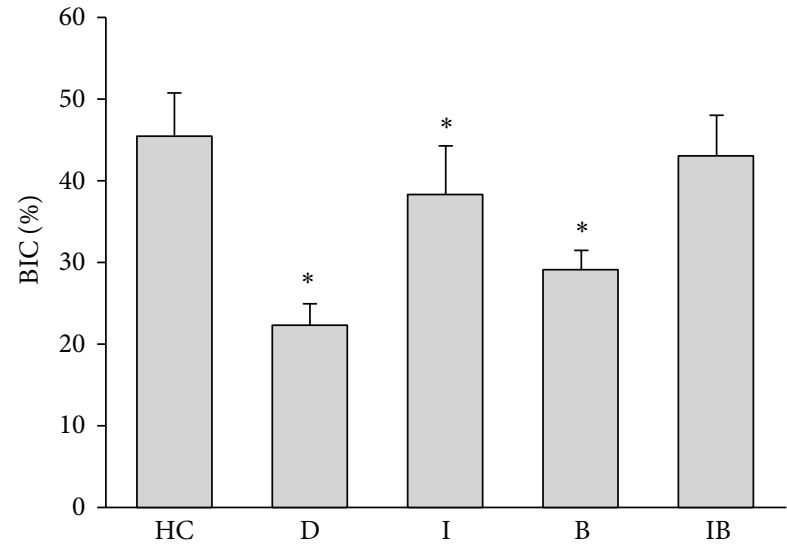

(b)

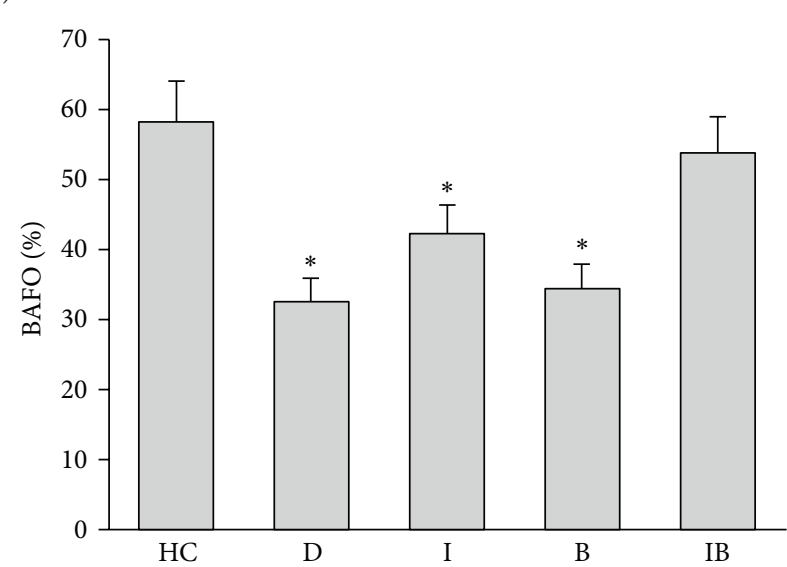

(c)

FIGURE 5: Histological images and quantitative analysis of implants 8 weeks after surgery by toluidine blue staining (10x). (a) HC: healthy control; D: nontreated diabetic; I: insulin-treated diabetic; B: berberine-treated diabetic; IB: berberine + insulin-treated diabetic. (b) Boneto-implant contact ratio. (c) Bone area fraction occupancy ratio. Data are expressed as mean $\pm \mathrm{SD}, n=10 .{ }^{*} P<0.05$, for healthy control rats versus others.

both insulin and AMPK signaling to increase glucose uptake, it could not alter the insulin secretion and synthesis therefore did not reverse total insulin reduction caused by destroyed pancreas beta cells [33]. This incomplete control of hyperglycemia may further explain weaker promotion of berberine on osseointegration compared with insulin. Interestingly, no weight gains were observed in Group B though PGLs were reduced, implying that berberine might have weaker or delayed regulation on body energy homeostasis compared to its effect on glucose modulation.

We further investigated the effect of berberine and insulin combined treatment on implant osseointegration and whether the effect is additive. As is shown in PGLs result, berberine together with insulin treatment successfully corrected PGLs after 4 weeks. Furthermore, values of BIC and BAFO in Group IB increased and were equivalent to those observed in Group HC. As ALP and BGP secreted by osteoblasts are associated with early and subsequent bone formation stages $[34,35]$, serum measurement of these two biomarkers in correlation with histological assessments of osseointegration can provide important information about the influence of the treatment on bone metabolism. The serum total ALP level in Groups B and IB was higher than that of Group D, while the difference was not significant $(P>$ 0.05). Interestingly, the correlation between serum ALP levels and localized osseointegration was not reflected. This might be due to the fact that alkaline phosphates are secreted by various sources including nonskeletal tissues, such as liver, intestine, and spleen. We also found significantly increased serum BGP levels in Groups I, B, and IB, with the highest value in Group IB. Taken together, the corrected PGLs, improved osseointegration, and highest levels of BGP in Group IB suggest that berberine in combination with insulin may lead to an additive or synergistic effect on glycemic control and bone activity. The improved osseointegration might be attributed to multiple factors mainly including corrected hyperglycemia, enhanced bone formation, and inhibited bone resorption: (1) abnormality in insulin receptor (InsR) is the major cause for the development of insulin resistance and type 2 diabetes mellitus. Berberine mimics insulin action and attenuates insulin resistance by increasing IR phosphorylation [15]. Therefore, when applied together, berberine and insulin may have additive effect in glucose uptake. (2) Berberine has potent antioxidant effects in reducing protein damage, DNA stand breaking, and inflammatory markers level in diabetic rats, which is beneficial for 
maintaining a suitable environment for bone healing [36, 37]. (3) Berberine decreases the level of bone loss by inhibiting osteoclasts formation and differentiation [22].

With the steady increase of diabetes patients, therapies that promote higher clinical efficiency and shorter rehabilitation duration are of great urgency in dental implant treatment. This study took advantage of the multiple functions of berberine in glucose homeostasis, insulin sensitizing, and bone anabolism, which are closely related to the promotion of bone formation in diabetic patients. We found that a combination of insulin and berberine (two existing drugs) seemed to be more effective than each applied alone, which may be because of their different and complementary mechanisms of action. Admittedly, differences of development and characterization could exist between the tibia and jaw. Further analysis at the cellular and molecular levels is also helpful to elucidate the intrinsic mechanism of action of the combination therapy. Also, modifying the chemistry of berberine to enhance its properties in glucose homeostasis and bone anabolism may also be encouraged.

\section{Conflict of Interests}

The authors declare that there is no conflict of interests regarding the publication of this paper.

\section{Acknowledgments}

This study was supported by a grant from the National Natural Science Foundation of China 81000455 (Chen Wenchuan) and Sichuan University. Supports provided by Drs. Ga Liao and Ning Ji of the State Key Laboratory of Oral Diseases at Sichuan University are greatly appreciated.

\section{References}

[1] P.-I. Brånemark, U. Breine, R. Adell, B. O. Hansson, J. Lindström, and A. Ohlsson, "Intra-osseous anchorage of dental prostheses. I. Experimental studies," Scandinavian Journal of Plastic and Reconstructive Surgery and Hand Surgery, vol. 3, no. 2, pp. 81-100, 1969.

[2] A. Mombelli and N. Cionca, "Systemic diseases affecting osseointegration therapy," Clinical Oral Implants Research, vol. 17, supplement 2, pp. 97-103, 2006.

[3] F. Marchand, A. Raskin, A. Dionnes-Hornes et al., "Dental implants and diabetes: conditions for success," Diabetes and Metabolism, vol. 38, no. 1, pp. 14-19, 2012.

[4] F. Wang, Y.-L. Song, D.-H. Li et al., "Type 2 diabetes mellitus impairs bone healing of dental implants in GK rats," Diabetes Research and Clinical Practice, vol. 88, no. 1, pp. e7-e9, 2010.

[5] M. A. Pfeffer, E. A. Burdmann, C.-Y. Chen et al., "A trial of darbepoetin alfa in type 2 diabetes and chronic kidney disease," New England Journal of Medicine, vol. 361, no. 21, pp. 2019-2032, 2009.

[6] J. H. DeVries, "Therapies for type 2 diabetes and coronary artery disease," The New England Journal of Medicine, vol. 361, no. 14, pp. 1408-1409, 2009.

[7] M. J. Tolosa, S. R. Chuguransky, C. Sedlinsky et al., "Insulindeficient diabetes-induced bone microarchitecture alterations are associated with a decrease in the osteogenic potential of bone marrow progenitor cells: preventive effects of metformin," Diabetes Research and Clinical Practice, vol. 101, no. 2, pp. 177186, 2013.

[8] F. Javed and G. E. Romanos, "Impact of diabetes mellitus and glycemic control on the osseointegration of dental implants: a systematic literature review," Journal of Periodontology, vol. 80, no. 11, pp. 1719-1730, 2009.

[9] R. Margonar, C. E. Sakakura, M. Holzhausen, M. T. Pepato, J. R. C. Alba, and J. E. Marcantonio, "The influence of diabetes mellitus and insulin therapy on biomechanical retention around dental implants: a study in rabbits," Implant Dentistry, vol. 12, no. 4, pp. 333-339, 2003.

[10] B. Wang, Y. Song, F. Wang et al., "Effects of local infiltration of insulin around titanium implants in diabetic rats," British Journal of Oral and Maxillofacial Surgery, vol. 49, no. 3, pp. 225229, 2011.

[11] S. Kotsovilis, I. K. Karoussis, and I. Fourmousis, "A comprehensive and critical review of dental implant placement in diabetic animals and patients," Clinical Oral Implants Research, vol. 17, no. 5, pp. 587-599, 2006.

[12] M. Eddouks, A. Bidi, B. El Bouhali, L. Hajji, and N. A. Zeggwagh, "Antidiabetic plants improving insulin sensitivity," Journal of Pharmacy and Pharmacology, vol. 66, no. 9, pp. 11971214, 2014.

[13] Y. Liu, L. Zhang, H. Song, and G. Ji, "Update on berberine in nonalcoholic fatty liver disease," Evidence-Based Complementary and Alternative Medicine, vol. 2013, Article ID 308134, 8 pages, 2013.

[14] Y. X. Ni, "Therapeutic effect of berberine on 60 patients with type II diabetes mellitus and experimental research," Zhong Xi Yi Jie He Za Zhi, vol. 8, no. 12, Article ID 707, pp. 711-713, 1988.

[15] C. Chen, Y. Zhang, and C. Huang, "Berberine inhibits PTP1B activity and mimics insulin action," Biochemical and Biophysical Research Communications, vol. 397, no. 3, pp. 543-547, 2010.

[16] A. Cok, C. Plaisier, M. J. Salie, D. S. Oram, J. Chenge, and L. L. Louters, "Berberine acutely activates the glucose transport activity of GLUT1," Biochimie, vol. 93, no. 7, pp. 1187-1192, 2011.

[17] P. Yi, F.-E. Lu, L.-J. Xu, G. Chen, H. Dong, and K.-F. Wang, "Berberine reverses free-fatty-acid-induced insulin resistance in 3T3-L1 adipocytes through targeting IKK $\beta$," World Journal of Gastroenterology, vol. 14, no. 6, pp. 876-883, 2008.

[18] Y. Chen, Y. Wang, J. Zhang, C. Sun, and A. Lopez, "Berberine improves glucose homeostasis in streptozotocin-induced diabetic rats in association with multiple factors of insulin resistance," ISRN Endocrinology, vol. 2011, Article ID 519371, 8 pages, 2011.

[19] J. Yin, H. Xing, and J. Ye, "Efficacy of berberine in patients with type 2 diabetes mellitus," Metabolism: Clinical and Experimental, vol. 57, no. 5, pp. 712-717, 2008.

[20] K. Tao, D. Xiao, J. Weng, A. Xiong, B. Kang, and H. Zeng, "Berberine promotes bone marrow-derived mesenchymal stem cells osteogenic differentiation via canonical Wnt/ $\beta$-catenin signaling pathway," Toxicology Letters, vol. 240, no. 1, pp. 68-80, 2016.

[21] H. W. Lee, J. H. Suh, H. N. Kim et al., "Berberine promotes osteoblast differentiation by Runx 2 activation with p38 MAPK," Journal of Bone and Mineral Research, vol. 23, no. 8, pp. 12271237, 2008.

[22] P. Wei, L. Jiao, L.-P. Qin, F. Yan, T. Han, and Q.-Y. Zhang, "Effects of berberine on differentiation and bone resorption of 
osteoclasts derived from rat bone marrow cells," Zhong Xi Yi Jie He Xue Bao, vol. 7, no. 4, pp. 342-348, 2009.

[23] J.-P. Hu, K. Nishishita, E. Sakai et al., "Berberine inhibits RANKL-induced osteoclast formation and survival through suppressing the NF-kappaB and Akt pathways," European Journal of Pharmacology, vol. 580, no. 1-2, pp. 70-79, 2008.

[24] D. Xu, W. Yang, C. Zhou, Y. Liu, and B. Xu, "Preventive effects of berberine on glucocorticoid-induced osteoporosis in rats," Planta Medica, vol. 76, no. 16, pp. 1809-1813, 2010.

[25] H. Li, T. Miyahara, Y. Tezuka, Q. Le Tran, H. Seto, and S. Kadota, "Effect of berberine on bone mineral density in SAMP6 as a senile osteoporosis model," Biological and Pharmaceutical Bulletin, vol. 26, no. 1, pp. 110-111, 2003.

[26] K. Srinivasan, B. Viswanad, L. Asrat, C. L. Kaul, and P. Ramarao, "Combination of high-fat diet-fed and low-dose streptozotocintreated rat: a model for type 2 diabetes and pharmacological screening," Pharmacological Research, vol. 52, no. 4, pp. 313-320, 2005.

[27] R. P. Guimarães, P. A. D. de Oliveira, and A. M. S. D. Oliveira, "Effects of induced diabetes and the administration of aminoguanidine in the biomechanical retention of implants: a study in rats," Journal of Periodontal Research, vol. 46, no. 6, pp. 691-696, 2011.

[28] J. T. Siqueira, S. C. Cavalher-Machado, V. E. Arana-Chavez, and P. Sannomiya, "Bone formation around titanium implants in the rat tibia: role of insulin," Implant dentistry, vol. 12, no. 3, pp. 242251, 2003.

[29] T. Lu, Y. Liang, J. Song, L. Xie, G. J. Wang, and X. D. Liu, "Simultaneous determination of berberine and palmatine in rat plasma by HPLC-ESI-MS after oral administration of traditional Chinese medicinal preparation Huang-Lian-Jie-Du decoction and the pharmacokinetic application of the method," Journal of Pharmaceutical and Biomedical Analysis, vol. 40, no. 5, pp. 1218-1224, 2006.

[30] Y. Gao, E. Luo, J. Hu, J. Xue, S. Zhu, and J. Li, "Effect of combined local treatment with zoledronic acid and basic fibroblast growth factor on implant fixation in ovariectomized rats," Bone, vol. 44, no. 2, pp. 225-232, 2009.

[31] E. C. McNay, J. A. Teske, C. M. Kotz et al., "Long-term, intermittent, insulin-induced hypoglycemia produces marked obesity without hyperphagia or insulin resistance: a model for weight gain with intensive insulin therapy," American Journal of Physiology-Endocrinology and Metabolism, vol. 304, no. 2, pp. E131-E138, 2013.

[32] S. A. Ross, H. D. Tildesley, and J. Ashkenas, "Barriers to effective insulin treatment: the persistence of poor glycemic control in type 2 diabetes," Current Medical Research and Opinion, vol. 27, supplement 3, pp. 13-20, 2011.

[33] J. Zhou, S. Zhou, J. Tang et al., "Protective effect of berberine on beta cells in streptozotocin- and high-carbohydrate/high-fat diet-induced diabetic rats," European Journal of Pharmacology, vol. 606, no. 1-3, pp. 262-268, 2009.

[34] Z. Du, J. Chen, F. Yan, N. Doan, S. Ivanovski, and Y. Xiao, "Serum bone formation marker correlation with improved osseointegration in osteoporotic rats treated with simvastatin," Clinical Oral Implants Research, vol. 24, no. 4, pp. 422-427, 2013.

[35] F. J. de Paula and C. J. Rosen, "Bone remodeling and energy metabolism: new perspectives," Bone Research, vol. 1, no. 1, pp. 72-84, 2013.

[36] W. Liu, P. Liu, S. Tao et al., "Berberine inhibits aldose reductase and oxidative stress in rat mesangial cells cultured under high glucose," Archives of Biochemistry and Biophysics, vol. 475, no. 2, pp. 128-134, 2008.

[37] J.-Y. Zhou and S.-W. Zhou, "Protective effect of berberine on antioxidant enzymes and positive transcription elongation factor b expression in diabetic rat liver," Fitoterapia, vol. 82, no. 2, pp. 184-189, 2011 


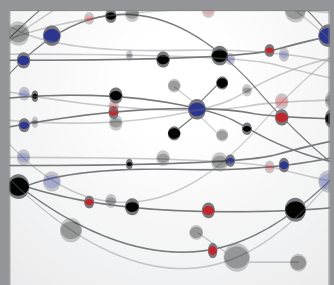

The Scientific World Journal
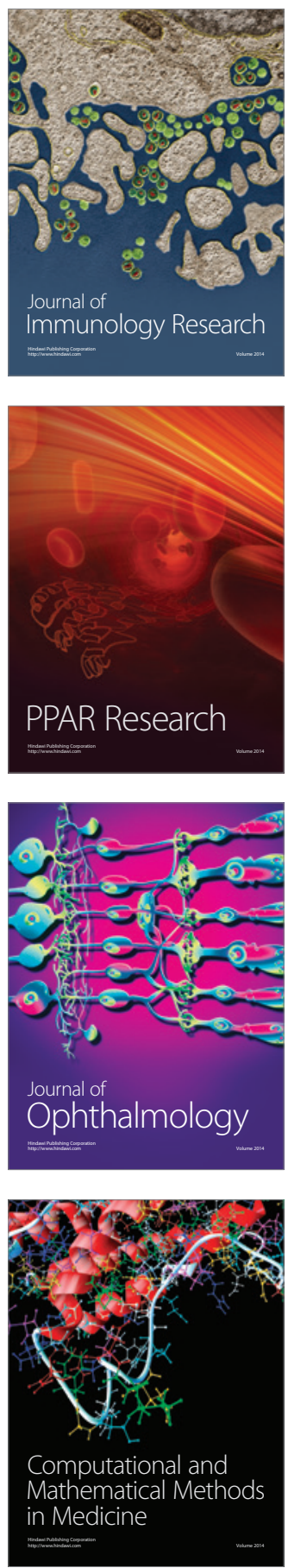

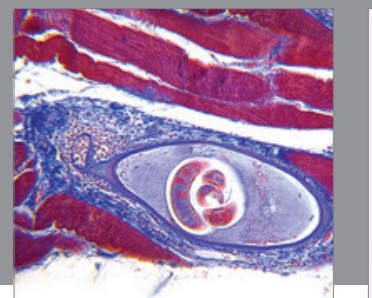

Gastroenterology

Research and Practice
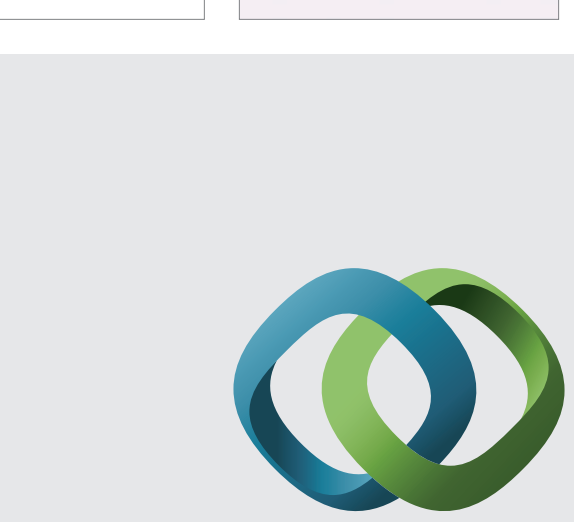

\section{Hindawi}

Submit your manuscripts at

http://www.hindawi.com
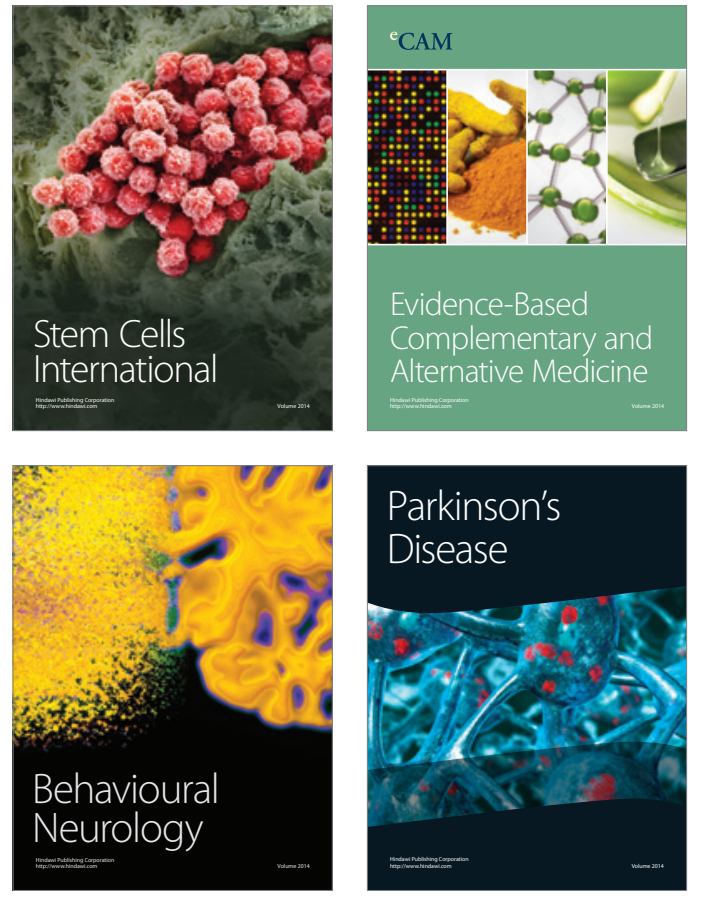
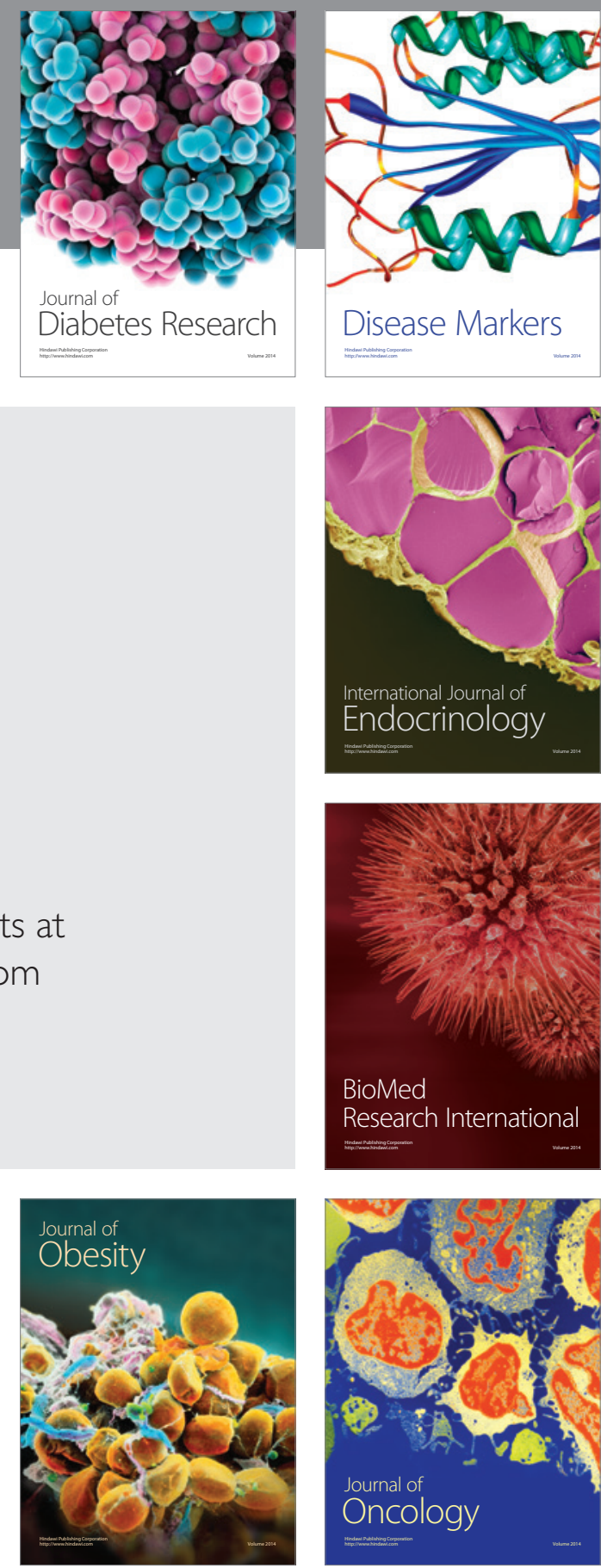

Disease Markers
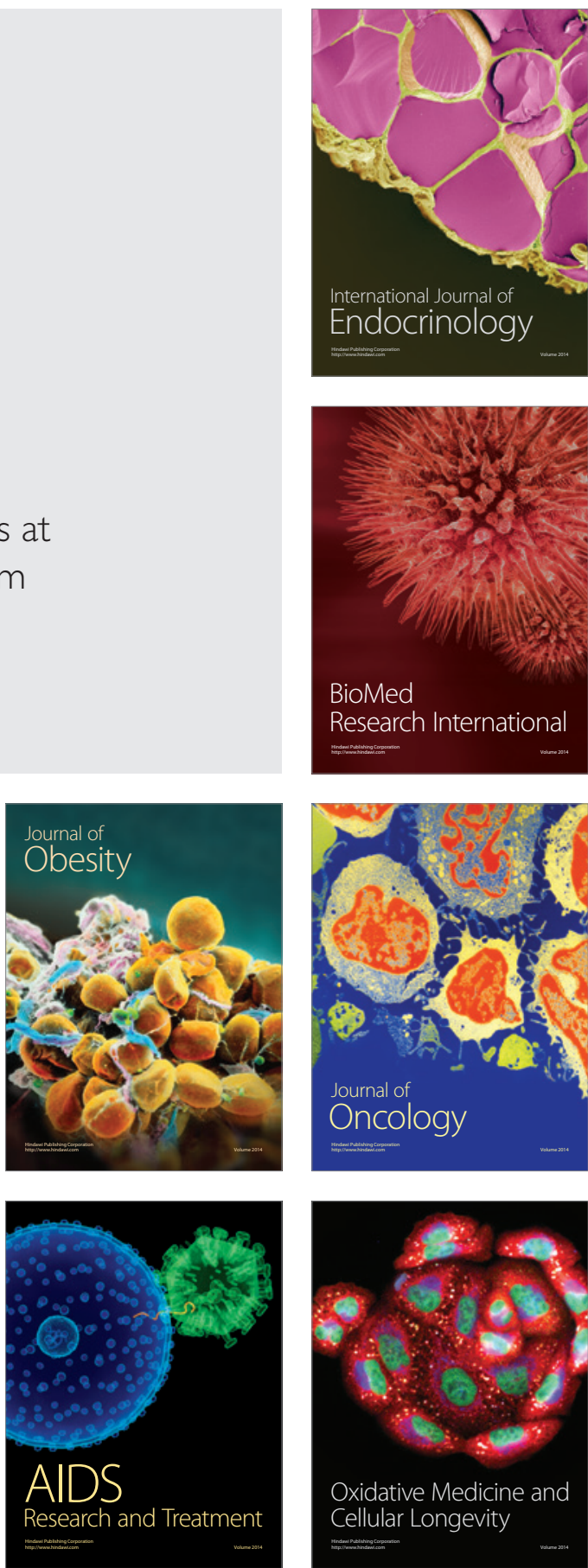